\title{
(HALF) A LECTURE ON D-BRANES
}

\author{
C. BACHAS * \\ Centre de Physique Théorique, Ecole Polytechnique, \\ 91128 Palaiseau, FRANCE \\ email: bachas@orphee.polytechnique.fr
}

This is a concise foreword to, rather than a review of, D-brane physics.

\section{Introduction}

In their early days dual models were proposed as a theory of hadronic interactions. Mesons are described by open strings, and in this context it is natural to consider heavy quarks at their endpoints. D-branes are generalized heavy quarks, which in the context of a fundamental theory acquire, both poetically and literally, some completely novel dimensions. First, in contrast to the quarks of QCD, D-branes are believed to be intrinsic to the fundamental theory: without them there could be no web of dualities, 2 , 3 holding all consistent superstrings inside a unique though still mysterious $(\mathcal{M}$ or $\mathcal{F}$ ?) structure. Second, being non-perturbative excitations of gravity, D-branes are in the process of modifying drastically our thinking about quantum space-time. The fascinating interplay of supersymmetric gauge theory and geometry has shed some surprising new light on both. Among all the exciting recent developments, this could prove the lesson whose consequences are most far-reaching.

This lecture is based on Polchinski's original paper 4 that recognized Dbranes as genuine non-perturbative excitations of string theory. It is not a review, but rather a concise foreword to the already large literature on the many different facets of the subject: duality checks, black-hole entropy, Dbrane scattering and D-brane probes, orientifold compactifications. Some of these topics are covered in other recent reviews $4,6,6,8,8,10,11,12$, but the story is unfolding fast and it is still unclear where it will take us.

${ }^{*}$ Lecture at the Workshop on Gauge Theories, Applied Supersymmetry and Quantum Gravity, Imperial College, London (July 1996), and at the Institut d' Eté, Ecole Normale Supérieure, Paris (August 1996). 


\section{Ramond-Ramond fields}

\subsection{Chiral Bispinors}

With the exception of the heterotic string, all other consistent string theories contain in their spectrum antisymmetric tensor fields coming from the Ramond-Ramond sector. This is the case for the type-IIa and IIb superstrings, as well as for the type-I theory whose closed-string states are a subset of those of type-IIb. The spectrum of a type-II theory is obtained as a tensor product of a left- and a right-moving world-sheet sector, each of which contains at the massless level a 10d vector and a 10d Weyl-Majorana spinor. This is depicted figuratively as follows:

$$
(|\mu\rangle \oplus|\alpha\rangle)_{\text {left }} \otimes(|\nu\rangle \oplus|\beta\rangle)_{\text {right }}
$$

where $\mu, \nu=0, \ldots, 9$ and $\alpha, \beta=1, \ldots, 16$ are respectively vector and spinor indices. Bosonic fields thus include a two-index tensor, which can be decomposed into symmetric traceless, trace, and antisymmetric parts: these are the usual fluctuations of the graviton $\left(G_{\mu \nu}\right)$, dilaton $(\Phi)$ and Neveu-Schwarz Neveu-Schwarz antisymmetric tensor $\left(B_{\mu \nu}\right)$. In addition massless bosonic fields include a Ramond-Ramond bispinor $F_{\alpha \beta}$, defined as the polarization in the corresponding vertex operator

$$
V_{R R} \sim F_{\alpha \beta}(p) \int d^{2} \zeta S^{\alpha}\left(i \Gamma^{0} \bar{S}\right)^{\beta} e^{i p X}
$$

Here $S, \bar{S}$ are the left and right fermionic emission vertices, $p$ the $10 \mathrm{~d}$ momentum, and the inclusion of the $\Gamma^{0}$ ensures that under a Lorentz transformation $F$ transforms by a similarity transformation. Such bispinors can be decomposed in a complete basis of all gamma-matrix antisymmetric products a

$$
F_{\alpha \beta}=\sum_{k=0}^{10} \frac{i^{k}}{k !} F_{\mu_{1} \ldots \mu_{k}}\left(\Gamma^{\mu_{1} \ldots \mu_{k}}\right)_{\alpha \beta}
$$

where

$$
\Gamma^{\mu_{1} \ldots \mu_{k}} \equiv \frac{1}{k !} \Gamma^{\left[\mu_{1}\right.} \ldots \Gamma^{\left.\mu_{k}\right]}
$$

${ }^{a}$ I follow the conventions of Green, Schwarz and Witten 13 : the 10d gamma matrices are purely imaginary and obey the algebra $\left\{\Gamma^{\mu}, \Gamma^{\nu}\right\}=-2 \eta^{\mu \nu}$ with metric signature $(-+\ldots+)$. Factors of $i$ are inserted appropriately so that the bispinor and its tensor components are all real. The chirality operator is $\Gamma_{11}=\Gamma_{0} \Gamma_{1} \ldots \Gamma_{9}$, and the Levi-Civita tensor $\epsilon^{01 \ldots 9}=1$. 
and the $k=0$ term stands by convention for the identity in spinor space. As a result the Ramond Ramond massless fields are a collection of antisymmetric Lorentz tensors.

These tensor components are not all independent, because the bispinor field has definite chirality projections

$$
\Gamma_{11} F= \pm F \Gamma_{11}=F
$$

The choice of sign distinguishes between the type-IIa and type-IIb models, for which the two spinors are, respectively, of opposite or same chirality in ten dimensions. Taking into account our definition of the bispinor field, we see that type-IIa corresponds to the sign plus and type-IIb to the sign minus. To rewrite eqs.(2.4) in terms of the tensor fields we need the gamma identities

$$
\begin{aligned}
\Gamma_{11} \Gamma^{\mu_{1} \ldots \mu_{k}} & =\frac{(-)^{\left[\frac{k}{2}\right]}}{(10-k) !} \epsilon^{\mu_{1} \ldots \mu_{10}} \Gamma_{\mu_{k+1} \ldots \mu_{10}} \\
\Gamma^{\mu_{1} \ldots \mu_{k}} \Gamma_{11} & =\frac{(-)^{\left[\frac{k+1}{2}\right]}}{(10-k) !} \epsilon^{\mu_{1} \ldots \mu_{10}} \Gamma_{\mu_{k+1} \ldots \mu_{10}}
\end{aligned}
$$

with $[x]$ denoting the integer part of $x$. It follows easily that only even- $k$ (odd$k$ ) terms are allowed in the type-IIa (type-IIb) case, and that furthermore all antisymmetric fields obey the duality relations

$$
F^{\mu_{1} \ldots \mu_{k}}=\frac{(-)^{\left[\frac{k+1}{2}\right]}}{(10-k) !} \epsilon^{\mu_{1} \ldots \mu_{10}} F_{\mu_{k+1} \ldots \mu_{10}}
$$

We write these relations in short-hand form as $F_{(k)}= \pm{ }^{*} F_{(10-k)}$. As a check note that the type-IIa theory has independent tensors with $k=0,2$ and 4 indices, while the type-IIb theory has $k=1,3$ and a self-dual $k=5$ tensor. The number of independent tensor components adds up in both cases to $16 \times 16=256$ :

$$
\begin{array}{ll}
\underline{\text { type }- \text { IIa : }} & 1+\frac{10 \times 9}{2 !}+\frac{10 \times 9 \times 8 \times 7}{4 !}=256, \\
\text { type }- \text { IIb : } & 10+\frac{10 \times 9 \times 8}{3 !}+\frac{10 \times 9 \times 8 \times 7 \times 6}{2 \times 5 !}=256,
\end{array}
$$

which is precisely the number of components of a bispinor. 


\subsection{Free Field Equations}

The mass-shell or super-Virasoro conditions for the vertex operator (2.1) imply that the bispinor field obeys two massless Dirac equations

$$
\left(p_{\mu} \Gamma^{\mu}\right) F=F\left(p_{\mu} \Gamma^{\mu}\right)=0 .
$$

To convert these to equations for the tensors we use the gamma identities

$$
\begin{aligned}
\Gamma^{\mu} \Gamma^{\nu_{1} \ldots \nu_{k}} & =\Gamma^{\mu \nu_{1} \ldots \nu_{k}}-\frac{1}{(k-1) !} \eta^{\mu\left[\nu_{1}\right.} \Gamma^{\left.\nu_{2} \ldots \nu_{k}\right]} \\
\Gamma^{\nu_{1} \ldots \nu_{k}} \Gamma^{\mu} & =\Gamma^{\nu_{1} \ldots \nu_{k} \mu}-\frac{1}{(k-1) !} \eta^{\mu\left[\nu_{k}\right.} \Gamma^{\left.\nu_{1} \ldots \nu_{k-1}\right]}
\end{aligned}
$$

with square brackets denoting the alternating sum over all permutations of the enclosed indices. After some straightforward algebra one finds

$$
p^{[\mu} F^{\left.\nu_{1} \ldots \nu_{k}\right]}=p_{\mu} F^{\mu \nu_{2} \ldots \nu_{k}}=0
$$

which are the Bianchi identity and free massless equation for an antisymmetric tensor field strength. We may write these in economic form as

$$
d F=d^{*} F=0
$$

Solving the Bianchi identity locally allows us to express the $k$-index field strength as the exterior derivative of a $(k-1)$-form potential

$$
F_{\mu_{1} \ldots \mu_{k}}=\frac{1}{(k-1) !} \partial_{\left[\mu_{1}\right.} C_{\left.\mu_{2} \ldots \mu_{k}\right]},
$$

or in short-hand notation

$$
F_{(k)}=d C_{(k-1)} .
$$

Thus the type-IIa theory has a vector $\left(C^{\mu}\right)$ and a three-index tensor potential $\left(C^{\mu \nu \rho}\right)$, in addition to a constant non-propagating zero-form field strength $(F)$, while the type-IIb theory has a zero-form $(C)$, a two-form $\left(C^{\mu \nu}\right)$ and a four-form potential $\left(C^{\mu \nu \rho \sigma}\right)$, the latter with self-dual field strength. The number of physical transverse degrees of freedom adds up in both cases to $64=8 \times 8$ :

$$
\begin{array}{ll}
\underline{\text { type }- \text { IIa : }} & 8+\frac{8 \times 7 \times 6}{3 !}=64, \\
\text { type - IIb : } & 1+\frac{8 \times 7}{2 !}+\frac{8 \times 7 \times 6 \times 5}{2 \times 4 !}=64,
\end{array}
$$

which is precisely the number of physical components of a bispinor. 


\subsection{No RR Charges in Perturbation Theory}

A $(p+1)$-form potential couples naturally to a $p$-brane, i.e. an extended object with $p$ spatial dimensions. The coupling is the integral of the form over the $(p+1)$-dimensional world-volume of the brane:

$$
\int_{\substack{\text { world } \\ \text { vol }}} C_{(p+1)} \equiv \int d^{p+1} \zeta C_{\mu_{1} \ldots \mu_{p+1}}(X) \partial_{1} X^{\mu_{1}} \ldots \partial_{p+1} X^{\mu_{p+1}}
$$

where $X^{\mu}\left(\zeta^{\alpha}\right)$ are the target-space coordinates of the brane 1 . For example the elementary charge for a vector potential is a point-particle or 0-brane, that for a two-index tensor is a 1-brane or string, and so on down the line. In view of the duality relation (2.7), $p$-branes and $(6-p)$-branes are electric-magnetic duals of each other in ten dimensions.

Now string perturbation theory contains no such elementary RR charges: to start with it has no branes other than the fundamental strings, which could at most couple to the RR two-index tensor. That even this doesn't happen follows from the fact that a trilinear coupling $\left\langle s\left|C_{(k)}\right| s\right\rangle$, corresponding to the emission of a RR $k$-form from any given string state, would violate the conservation laws of separate left and right fermion numbers, which are valid to all orders in the genus expansion. Note also that the $R R$ vertex operator involves the field strength rather than the potential, and that the field equations and Bianchi identities entered in a completely symmetric way: this duality of perturbation theory would have been destroyed by the presence of either electric or magnetic charges.

Most non-pertubative dualities require, on the other hand, the existence of such elementary charges. Two of these dualities involve the strong-coupling limits of the type-IIa and type-IIb theories in ten dimensions 1 , 3. The former is conjectured to be $\mathcal{M}$ theory, whose low-energy limit is 11d supergravity. Indeed, the massless RR and NSNS fields of the IIa theory can be obtained by dimensional reduction from eleven dimensions:

$$
G_{M N} \rightarrow G_{\mu \nu}, C_{\mu}, \Phi ; \quad C_{M N R} \rightarrow C_{\mu \nu \rho}, B_{\mu \nu}
$$

with $M, N, R=0, \ldots, 10$. In $11 \mathrm{~d}$ supergravity there are however also KaluzaKlein states carrying charge under the off-diagonal metric components $A_{\mu}$. Since this is a RR field in type-IIa theory, duality requires the existence of non-perturbative 0-brane charges. Likewise the type-IIb theory has a $\mathrm{SL}(2, \mathrm{Z})$ self-duality under which the dilaton and RR scalar $C$ transform together as

\footnotetext{
${ }^{b}$ Lower-case Greek letters label both the world-volume coordinates and the space-time spinors. Put in context, this should hopefully cause no confusion.
} 
a complex modulus, while the two 2-index antisymmetric forms $\left(B_{\mu \nu}, C_{\mu \nu}\right)$ transform as a doublet 4,15 . Since fundamental strings couple as elementary charges to $B_{\mu \nu}$, this duality requires the existence of non-perturbative 1-branes that are likewise charged under $C_{\mu \nu}$.

Higher p-branes fit similarly in the conjectured web of dualities. For instance type-IIa theory compactified to six dimensions on a $K_{3}$ surface is expected to be dual to the heterotic string compactified on a four-torus 2 目. The latter has extended gauge symmetry at special points of the Narain moduli space. On the type-IIa side these charged massless gauge bosons can only come from 2-branes wrapping around shrinking 2-cycles of the K3 surface 1 , 17, 10 . A similar phenomenon occurs for Calabi-Yau compactifications: the effective low-energy Lagrangian of Ramond-Ramond fields has a logarithmic singularity at special (conifold) points in the Calabi-Yau moduli space. This can be understood as due to 3-branes wrapping argund shrinking 3-cycles, and thus becoming massless at these special points $16,28$.

Early efforts consisted in describing all these pxotic states as (singular) solutions of the effective low-energy supergravity 1 . Following Polchinski's work it has become, however, clear that they admit a much simpler, exact and unified description as allowed endpoints for open strings, or D-branes.

\section{$3 \quad \mathrm{D}$ (irichlet) branes}

\subsection{Definition}

The bosonic part of the world-sheet action for a free string, in flat space-time and in the conformal gauge, reads

$$
S_{2 d}=\int_{\mathcal{M}} \frac{d^{2} \zeta}{4 \pi \alpha^{\prime}} \partial_{\alpha} X^{\mu} \partial^{\alpha} X_{\mu}
$$

with $\mathcal{M}$ some generic surface with boundary. For its variation

$$
\delta S_{2 d}=-\int_{\mathcal{M}} \frac{d^{2} \zeta}{2 \pi \alpha^{\prime}} \delta X^{\mu} \partial_{\alpha} \partial^{\alpha} X_{\mu}+\int_{\partial \mathcal{M}} \frac{d \zeta^{\alpha}}{2 \pi \alpha^{\prime}} \delta X^{\mu} \varepsilon_{\alpha \beta} \partial^{\beta} X_{\mu}
$$

to vanish, the $X^{\mu}$ must be harmonic functions on the world sheet, and either of the following two conditions must hold on the boundary

$$
\begin{array}{rlll}
\partial_{\perp} X_{\mu} & =0 & & \text { (Neumann), } \\
\text { or } \quad \delta X^{\mu} & =0 & \text { (Dirichlet). }
\end{array}
$$

Neumann conditions respect Poincaré invariance and are hence momentumconserving. Dirichlet conditions on the other hand represent defects in spacetime. They were studied in the past in various guises, for instance as sources 
for partonic behaviour in string theory 19 , as heavy-quark endpoints 20 , and as T-dual forms of open-string compactifications 21, 22 , but their status of nonperturbative states of string theory was not fully appreciated in these early studies.

A static extended defect with $p$ spatial dimensions is described by the boundary conditions

$$
\partial_{\perp} X^{0,1, . ., p}=X^{p+1, . ., 9}=0,
$$

that force open strings to move on a $(p+1)$-dimensional (world-volume) hyperplane. Since open strings do not propagate in the bulk in type-II theory, their presence is intimately-tied to the existence of the defect which we will refer to as $\mathrm{D}(\mathrm{p})$-brane. The $\mathrm{D}(\mathrm{p})$-brane is characterized by a tension $T_{(p)}$, and charge density under the Ramond-Ramond $(p+1)$-form $\mu_{(p)}$, defined through the effective world-volume action

$$
S_{\substack{\text { world } \\ \text { vol }}}=T_{(p)} \int d^{p+1} \zeta e^{-\Phi / 2} \sqrt{\left|\operatorname{det} \hat{G}_{\alpha \beta}\right|}+\mu_{(p)} \int d^{p+1} \zeta C_{(p+1)},
$$

where

$$
\hat{G}_{\alpha \beta}=G^{\mu \nu} \partial_{\alpha} X_{\mu} \partial_{\beta} X_{\nu}
$$

is the induced world-volume metric. The massless closed strings coupling to the D-brane have themselves a bulk action 13

$$
S_{\text {bulk }}=-\frac{1}{2 \kappa_{(10)}^{2}} \int d^{10} x \sqrt{-G}\left[e^{-\Phi}\left(R-d \Phi^{2}+\frac{1}{12} d B^{2}\right)+\sum \frac{1}{2 k !} F_{(k)}^{2}\right]
$$

Here $k=0,2,4$ for type-IIa theory, $k=1,3$ for type-IIb, while for the selfdual $F_{(5)}$ there is no covariant action we may write down. Notice that the bulk Lagrangian coming from the sphere diagram is multiplied by the usual factor $e^{-\Phi}$, while the world-volume Lagrangian coming from the disk diagram is multiplied by $e^{-\Phi / 2}$. These factors were absorbed in the Ramond-Ramond fields through a rescaling

$$
C_{(p+1)} \rightarrow e^{\Phi / 2} C_{(p+1)} .
$$

A carefull analysis shows indeed that it is the field strength of rescaled potentials that satisfies the usual Bianchi identity and Maxwell equation when the dilaton varies 23 .

We will now calculate the values of $T_{(p)}$ and $\mu_{(p)}$ and check that they are compatible with supersymmetry and with the (minimal) charge-quantization condition. The charge density and tension could be extracted in principle 
from one-point functions on the disk. Following Polchinski 1 we will however prefer to extract them from the interaction energy between two parallel identical D-branes. This way of thinking avoids the technicalities of normalizing vertex operators correctly, and extends naturally to the study of D-brane dynamics $24,25$.

\subsection{Static Force: Field-Theory Calculation}

Viewed as solitons of 10d supergravity, two D-branes interact by exchanging gravitons, dilatons and antisymmetric tensors. This is a good approximation, provided their separation $r$ is large compared to the string scale, in which case the effective actions, eqs. (3.6-8) can be trusted. To decouple the propagators of the graviton and dilaton, we pass to the Einstein metric

$$
g_{\mu \nu}=e^{-\Phi / 4} G_{\mu \nu},
$$

in terms of which the effective actions take the form

$$
\begin{aligned}
S_{b u l k}=-\frac{1}{2 \kappa_{(10)}^{2}} & \int d^{10} x \sqrt{-g}\left[R+\frac{1}{8} d \Phi^{2}+\frac{1}{12} e^{-\Phi / 2} d B^{2}\right. \\
& \left.+\sum \frac{1}{2(p+2) !} e^{(3-p) \Phi / 4} d C_{(p+1)}^{2}\right]
\end{aligned}
$$

and

$$
S_{\text {world }}^{\text {vol }}=T_{(p)} \int d^{p+1} \zeta e^{(p-3) \Phi / 8} \sqrt{\left|\operatorname{det} \hat{g}_{\alpha \beta}\right|}+\mu_{(p)} \int d^{p+1} \zeta C_{(p+1)} .
$$

To leading order in the coupling constant the interaction energy comes from the exchange of a single graviton, dilaton or Ramond-Ramond field, and reads

$$
\mathcal{E}(r) \mathcal{T}=-2 \kappa_{(10)}^{2} \int d^{10} x \int d^{10} \tilde{x}\left[4 j_{\Phi} \Delta \tilde{j}_{\Phi}-j_{C} \Delta \tilde{j}_{C}+T_{\mu \nu} \Delta^{\mu \nu, \rho \tau} \tilde{T}_{\rho \tau}\right]
$$

Here $j_{\Phi}, j_{C}$ and $T_{\mu \nu}$ are the sources for the dilaton, RR form and graviton obtained by linearizing the world-volume action for one of the branes, while the tilde quantities refer to the other. $\Delta$ and $\Delta^{\mu \nu, \rho \tau}$ are the scalar and the graviton propagators in 10 dimensions, evaluated at the argument $(x-\tilde{x})$, and $\mathcal{T}$ the total interaction time. To simplify notation, and since only one component of $C_{(p+1)}$ couples to a static planar $\mathrm{D}(\mathrm{p})$-brane, we have dropped the obvious tensor structure of the RR field.

For such a static planar defect the sources take the simple form

$$
\begin{aligned}
& j_{\Phi}=\frac{p-3}{8} T_{(p)} \delta\left(x^{\perp}\right) \\
& j_{C}=\mu_{(p)} \delta\left(x^{\perp}\right)
\end{aligned}
$$




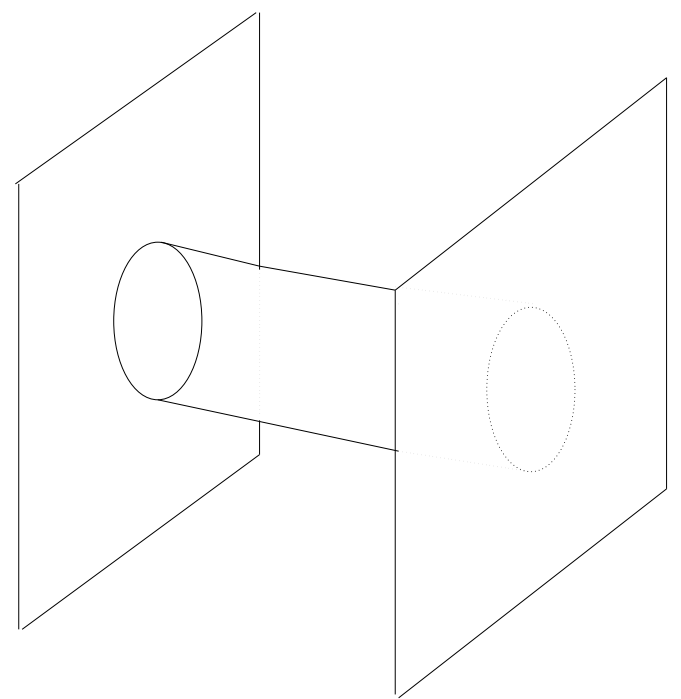

Figure 1: Two D-branes interacting through the exchange of a closed string. The diagram has a dual interpretation as Casimir force due to vacuum fluctuations of open strings.

$$
T_{\mu \nu}=\frac{1}{2} T_{(p)} \delta\left(x^{\perp}\right) \times\left\{\begin{array}{lr}
\eta_{\mu \nu} & \text { if } \mu, \nu \leq p \\
0 & \text { otherwise }
\end{array}\right.
$$

where the $\delta$-function localizes the defect in transverse space. The graviton propagator in the De Donder gauge and in $d$ dimensions reads 26

$$
\Delta_{(d)}^{\mu \nu, \rho \tau}=\left(\eta^{\mu \rho} \eta^{\nu \tau}+\eta^{\mu \tau} \eta^{\nu \rho}-\frac{2}{d-2} \eta^{\mu \nu} \eta^{\rho \tau}\right) \Delta_{(d)},
$$

where

$$
\Delta_{(d)}(x)=\int \frac{d^{d} p}{(2 \pi)^{d}} \frac{e^{i p x}}{p^{2}} .
$$

Putting all this together and doing some straightforward algebra we obtain

$$
\mathcal{E}(r)=2 V_{(p)} \kappa_{(10)}^{2}\left[\mu_{(p)}^{2}-T_{(p)}^{2}\right] \Delta_{(9-p)}^{E}(r)
$$

where $V_{(p)}$ is the (regularized) p-brane volume and $\Delta_{(9-p)}^{E}(r)$ is the (Euclidean) scalar propagator in $(9-p)$ transverse dimensions. The net force is as should be expected the difference between $\mathrm{RR}$ repulsion and gravitational plus dilaton attraction. 


\subsection{Static Force: String Calculation}

The exchange of all closed-string modes, including the massless graviton, dilaton and $\mathrm{RR}(p+1)$-form, is given by the cylinder diagram of figure 1. Considered, however, as an annulus, this same diagram also admits a dual and, from the field-theory point of view, surprising interpretation: the two D-branes interact by modifying the vacuum fluctuations of (stretched) open strings, in the same way that two superconducting plates attract by modifying the vacuum fluctuations of the photon field. It is this simple-minded duality which may. as we will see below, revolutionize our thinking about space-time.

The one-loop vacuum energy of open strings reads

$$
\begin{gathered}
\mathcal{E}(r)=-\frac{V^{(p)}}{2} \int \frac{d^{p+1} k}{(2 \pi)^{p+1}} \int_{0}^{\infty} \frac{d t}{t} \operatorname{Str} e^{-\pi t\left(k^{2}+M^{2}\right) / 2}= \\
=-2 \times \frac{V^{(p)}}{2} \int_{0}^{\infty} \frac{d t}{t}\left(2 \pi^{2} t\right)^{-(p+1) / 2} e^{-r^{2} t / 2 \pi} Z(t),
\end{gathered}
$$

where

$$
Z(t)=\frac{1}{2} \sum_{s=2,3,4}(-)^{s} \theta_{s}^{4}(i t / 2) \eta^{-12}(i t / 2)
$$

is the usual spin structure sum obtained by supertracing over open-string oscillator states, and we have set $\alpha^{\prime}=1 / 2$. Strings stretching between the two D-branes have at the $N$ th oscillator level a mass $M^{2}=(r / \pi)^{2}+2 N$, so that their vacuum fluctuations are modified when we separate the D-branes. The vacuum energy of open strings with both endpoints on the same defect is, on the other hand, $r$-independent and has been omitted. Notice also the (important) factor of 2 in front of the second line: it accounts for the two possible orientations of the stretched string,

The first remark concerning the above expression, is that it vanishes by the well-known $\theta$-function identity. Comparing with eq. (3.18) we conclude that

$$
T_{(p)}=\mu_{(p)},
$$

so that $\mathrm{RR}$ repulsion cancels exactly the gravitational and dilaton attraction. This is a consequence of space-time supersymmetry: the relation ( 3.21 ) between tension and charge is in fact the BPS condition for short multiplets of the $\mathrm{N}=8$ supersymmetry algebra in ten dimensions. Put differently, Dirichlet conditions corresponding to a single static defect identify left- and rightmoving spin fields on the boundary: half of the target-space supersymmetries 
are thus still linearly realized in perturbative string theory, i.e. they are unbroken in the presence of the D-brane. As is usual for BPS states, identical and parallel D-branes exert no net force on one another. This is analogous to the cancellation of Coulomb repulsion and Higgs-scalar attraction between the 'tHooft-Polyakov monopoles of $\mathrm{N}=4$ supersymmetric Yang-Mills.

To extract the actual value of $T_{(p)}$ we must separate in the diagram the exchange of RR and NS-NS closed-string states. These are characterized by world-sheet fermions which are periodic, respectively antiperiodic around the cylinder, so that they correspond to the $s=4$, respectively $s=2,3$ open-string spin structures. In the large-separation limit $(r \rightarrow \infty)$ we may furthermore expand the integrand near $t \sim 0$ :

$$
Z(t) \simeq(8-8) \times\left(\frac{t}{2}\right)^{4}+o\left(e^{-1 / t}\right) .
$$

Using the integral representation

$$
\Delta_{(d)}^{E}(r)=\frac{\pi}{2} \int_{0}^{\infty} d l\left(2 \pi^{2} l\right)^{-d / 2} e^{-r^{2} / 2 \pi l} .
$$

and restoring correct mass units we obtain

$$
\mathcal{E}(r)=V^{(p)}(1-1) 2 \pi\left(4 \pi^{2} \alpha^{\prime}\right)^{3-p} \Delta_{(9-p)}^{E}(r)+o\left(e^{-r / \sqrt{\alpha^{\prime}}}\right) .
$$

Comparing with the field-theory calculation we can finally extract the tension and charge-density of $\mathrm{D}(\mathrm{p})$-branes ,

$$
T_{(p)}^{2}=\mu_{(p)}^{2}=\frac{\pi}{\kappa_{(10)}^{2}}\left(4 \pi^{2} \alpha^{\prime}\right)^{3-p} .
$$

These are fixed as should be expected for intrinsic excitations of a fundamental theory. We will now see that they also pass the first non-trivial consistency condition.

\subsection{Charge Quantization}

Dirac's quantization condition for electric and magnetic charge has an analog for extended objects in higher dimensions 27. Consider a $\mathrm{D}(\mathrm{p})$-brane sitting at the origin, and integrate the field equation that follows from the action (3.6-8) over the transverse space. Using Stoke's theorem, one finds

$$
\int_{S_{(8-p)}} * F_{(p+2)}=2 \kappa_{(10)}^{2} \mu_{(p)}
$$




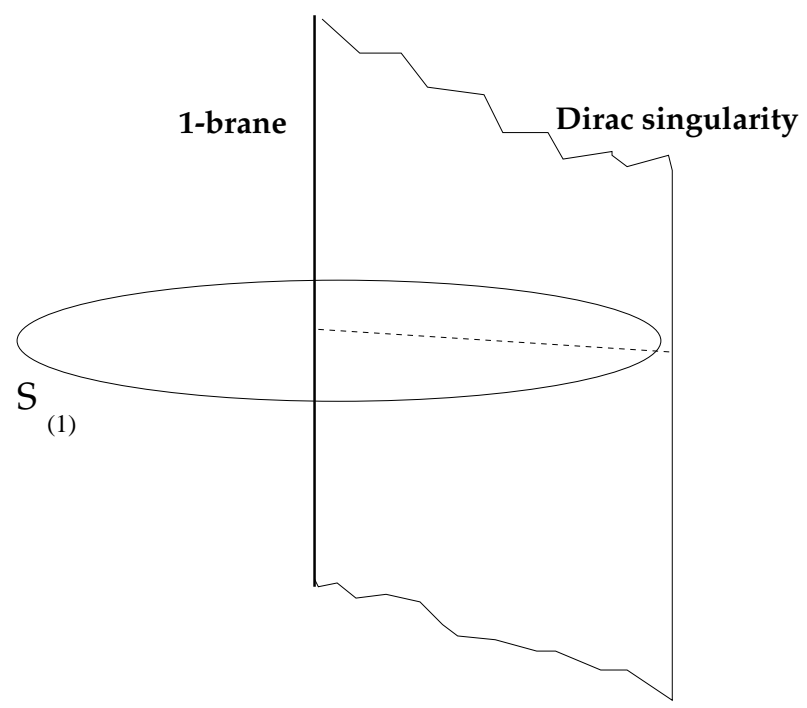

Figure 2: A 1-brane creates a 3-index "electric" field $F_{(3)}$. Electric flux in d=4 space-time dimensions is given by an integral of the dual vector over a 1-sphere. The magnetic potential is a scalar field with a discontinuity across the depicted Dirac sheet singularity.

where $S_{(8-p)}$ is a sphere around the defect as illustrated in figure 2. This is the analog of Gauss' law. Now Poincaré duality tells us that

$$
* F_{(p+2)}= \pm F_{(8-p)} \simeq \pm d C_{(7-p)},
$$

where the potential $C_{(7-p)}$ is not globally defined since the p-brane is a source in the Bianchi identity for $F_{(8-p)}$. Following Dirac we may define a smooth potential everywhere except along a singular (hyper)string cutting $S_{(8-p)}$ on a hypersphere $S_{(7-p)}$. This singularity is dangerous since a Bohm-Aharonov experiment involving (6-p)-branes might detect it. Indeed, the wave-function of a (6-p)-brane transported around the singuality picks a phase

$$
\begin{aligned}
\text { Phase }=\quad \mu_{(6-p)} \int_{S_{(7-p)}} C_{(7-p)}= \\
=\mu_{(6-p)} \int_{S_{(8-p)}} F_{(8-p)}= \pm 2 \kappa_{(10)}^{2} \mu_{(p)} \mu_{(6-p)} .
\end{aligned}
$$

For the (hyper)string not to be observed we must have

$$
\text { Phase }=2 \pi n \text {. }
$$


The D-brane charges found in the previous section satisfy this condition with $n=1$ ! The simple annulus diagram somehow knows about this non-perturbative consistency check $\mathrm{Q}$. Furthermore we have just learned that D-branes are the minimal RR charges allowed in the theory, so one may conjecture that there are no others.

\section{Gauge Theory and Geometry}

\subsection{Non-commutative Space-time}

Although the full dynamics of solitons cannot be separated from the field theory in which they belong, their low-energy dynamics can be approximated by quantum mechanics in the moduli-space of zero modes. For an extended pbrane defect, the zero modes give rise to massless world-volume fields and the quantum mechanics becomes a $(\mathrm{p}+1)$-dimensional field theory. Similar considerations apply to a $\mathrm{D}(\mathrm{p})$-brane: its perturbative excitations are described by a full-fledged open string theory, whose low-energy limit is an abelian supersymmetric Yang-Mills, dimensionnally-reduced from ten down to $(\mathrm{p}+1)$ dimensions,

$$
A^{\mu} \rightarrow A^{\alpha}\left(X^{\alpha}\right), A^{i}\left(X^{\alpha}\right) .
$$

I am using here a physical parametrization $X^{\alpha}=\zeta^{\alpha}(\alpha=0, . ., p)$, corresponding to a planar static background defect like the one of eqs. (3.5). The worldvolume scalars are proportional to the transverse coordinates of the brane

$$
X^{i}=2 \pi \alpha^{\prime} A^{i} \quad(i=p+1, . ., 9) .
$$

They are the Goldstone modes of broken translation invariance, forced by supersymmetry to be part of an entire vector multiplet.

That much can be in fact deduced within the context of $N=2$ supergravity in ten dimensions 9 . Where the role of string theory becomes important is in the presence of more than one D-branes. In addition to the massless vector multiplets describing the positions of each defect, there are now extra potentially-light fields corresponding to the open strings that stretch between the D-branes. These are the strings responsible for the Casimir force of the previous chapter. In the simplest case of two parallel D(p)-branes (figure 3), the ensuing low-energy field theory is a dimensionnally-reduced supersymmetric Yang-Mills with non-abelian gauge group 15

$$
U(2) \simeq U(1)_{c m} \times S U(2)_{\text {relative }} .
$$

This is indeed the low-energy limit of an oriented open-string theory with a Chan-Patton index $i=1,2$ labelling the two possible string endpoints. The 


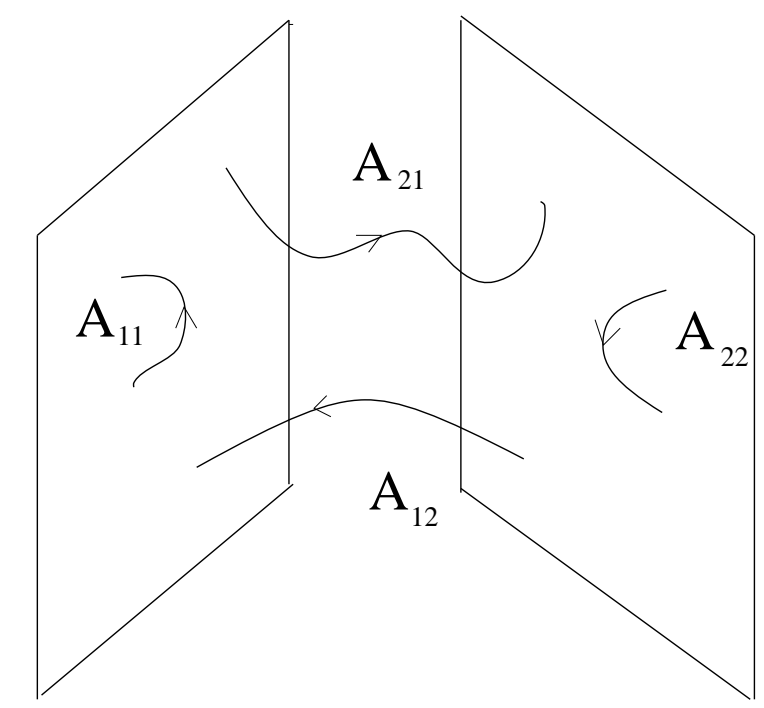

Figure 3: A D-brane sandwitch, and the four types of open strings giving rise to massless states in the coincidence limit.

abelian vector multiplet $A_{c m}=\left(A_{11}+A_{22}\right) / \sqrt{2}$ describes the dynamics of the center of mass, while the non-abelian $\mathrm{SU}(2)$ describes the reduced relative motion. At non-zero separation

$$
r=2 \pi \alpha^{\prime}\left|\left\langle A_{11}^{i}-A_{22}^{i}\right\rangle\right|,
$$

the non-abelian theory is in the spontaneously-broken phase: charged gauge bosons are stretched open strings that have a mass $M=r / 2 \pi \alpha^{\prime}$.

Figure 3 summarizes in itself much of the new insights brought by Dbranes. In a nutshell it teaches us that the short-distance structure of spacetime is intimately related to supersymmetric gauge field theory. The details of the theory depend of course on the precise configuration of D-branes, but the mapping between ultraviolet gravity and infrared gauge theory seems to be universally valide8. This fact underlies the successfull counting of the BeckensteinHawking entropy for extremal (BPS) configurations of D-branes 29, 11 . It is also the essential ingredient of the conjectured equivalence between infinitemomentum $\mathrm{M}$ theory, and $\mathrm{M}($ atrix)-model quantum mechanics 30 . 


\subsection{T-duality}

The dimensional reduction (4.1) suggests that the transverse D-brane coordinates are gauge fields in some invisible internal dimensions. The precise correspondence involves a (perturbative) T-duality transformation, which we will now make more explicit. T-duality is a local symmetry of the fundamental theory, that transforms both the background fields and the string excitations around them 11 . Recall that a closed-string coordinate along a compact (ninth) dimension reads

$$
X^{9}(z, \bar{z})=X^{9}(z)+\tilde{X}^{9}(\bar{z}),
$$

where

$$
\begin{aligned}
& X^{9}(z)=x^{9}-\frac{i}{2}\left(\frac{n_{9}}{2 R_{9}}+m_{9} R_{9}\right) \ln z+\frac{i}{2} \sum_{k \neq 0} \frac{a_{k}^{9}}{k} z^{-k} \\
& \tilde{X}^{9}(\bar{z})=\tilde{x}^{9}-\frac{i}{2}\left(\frac{n_{9}}{2 R_{9}}-m_{9} R_{9}\right) \ln \bar{z}+\frac{i}{2} \sum_{k \neq 0} \frac{\tilde{a}_{k}^{9}}{k} \bar{z}^{-k}
\end{aligned}
$$

Here $n_{9}$ and $m_{9}$ are the (integer in appropriate units) momentum and winding, and we are using radial world-sheet coordinates $z=e^{\tau+i \sigma}$. The simplest T-duality transformation inverts the radius, and interchanges winding with momentum:

$$
\tilde{X}^{9 \prime}=-\tilde{X}^{9}, \quad R_{9}^{\prime}=\frac{1}{2 R_{9}} \text { and }\left(n_{9}^{\prime}, m_{9}^{\prime}\right)=\left(m_{9}, n_{9}\right) .
$$

It is a hybrid parity operation, whose action is restricted to the antiholomorphic world-sheet sector.

The parity operator in spinor space is $-i \Gamma^{9} \Gamma_{11}$, so bispinor fields will transform as follows:

$$
F^{\prime}=-i F \Gamma^{9} \Gamma_{11} .
$$

Using the $\Gamma$-matrix identities of chapter 2 , we may rewrite this relation in component form,

$$
\begin{array}{r}
F_{\mu_{1} \ldots \mu_{k}}^{\prime}=-F_{9 \mu_{1} \ldots \mu_{k}} \\
F_{9 \mu_{1} \ldots \mu_{k}}^{\prime}=F_{\mu_{1} \ldots \mu_{k}}
\end{array}
$$

where $\mu_{i} \neq 9$ for all $i$. The duality exchanges even-k with odd-k antisymmetric forms, and hence also type IIa with type IIb backgrounds. Consistency requires that it also transform even-p to odd-p branes and vice versa.

To see how this comes about let us look at a $\mathrm{D}(\mathrm{p}+1)$-brane that wraps around the first $p$, as well as around the 9th dimension. We concentrate on 
the 9th coordinate of the open strings that live on the D-brane. Except for an extra factor 2 multiplying zero modes, because open strings are parametrized by $\sigma \in[0, \pi]$, the mode expansions (4.6) stay valid. The world-sheet is now the infinite strip, mapped in radial coordinates to the upper half complex plane. Imposing appropriate (Neumann) conditions on the boundary,

$$
X^{9}(z)=\tilde{X}^{9}(\bar{z}) \text { at } \operatorname{Im} z=0,
$$

identifies left and right oscillators and sets the winding number $m_{9}=0$. This is consistent with the fact that open strings move freely along the ninth dimension but cannot wind.

Now the action of a T-duality transformation changes the Neumann condition to Dirichlet,

$$
X^{9 \prime}(z)=-\tilde{X}^{9 \prime}(\bar{z}) \text { at } \operatorname{Im} z=0
$$

and momentum to winding 21 . The $(\mathrm{p}+1)$-brane is thus transformed to a $\mathrm{p}-$ brane: indeed, open strings cannot move along the 9th dimension any more, but since their endpoints are now attached they can wind. The inverse transformation is also true: a p-brane transverse to the 9th dimension becomes a $(\mathrm{p}+1)$-brane that wraps around it. All this is compatible with the transformation of the RR forms to which the D-branes couple. It is also compatiblet with the tension formula, eq. (3.25), if one takes into account the fact that a T-duality shifts the string coupling constant,

$$
\Phi^{\prime}=\Phi+\ln \left(\alpha^{\prime} / R^{2}\right) .
$$

Since T-duality is an isomorphism on the algebra of vertex operators, the full open string theory, and its low-energy limit in particular are unchanged. The gauge fields, which start out as functions of $X^{0, . ., p}$, and of $X^{9}$ or equivalently the conjugate momentum $n_{9}$, become after the transformation functions of the same first $(p+1)$ coordinates, and of the dual coordinate $X^{9 \prime}$ or equivalently the winding number $m_{9}^{\prime}$. The latter dependence can be ignored in the $R_{9} \rightarrow 0$ or $R_{9}^{\prime} \rightarrow \infty$ limit: this is precisely the limit of a dimensionally-reduced $(\mathrm{p}+1)$-brane, or of a dual p-brane transverse to an uncompactified ninth dimension.

\subsection{The Power of Lorentz Invariance}

To illustrate the power of this duality 24, 34, 36, consider the 0-brane of type-IIa theory performing some one-dimensional motion

$$
X^{0}=\tau, \quad X^{1}=f(\tau) .
$$


Its action, eq. (3.6), in flat space-time but non-zero background for the RR vector potential, reads

$$
S_{\text {world }}^{\text {line }}=T_{(0)} \int d \tau \sqrt{1-\dot{f}^{2}}+\mu_{(0)} \int d \tau\left(C_{0}+\dot{f} C_{1}\right)+\ldots
$$

where the dots stand for acceleration terms that we neglected. Let us now T-dualize the $X^{1}$ coordinate: this changes the 0-brane to a 1-brane, and the velocity to an electric field

$$
\mathcal{F}_{01}=\partial_{0} A_{1}=\dot{f} / 2 \pi \alpha^{\prime} .
$$

It also transforms the $\mathrm{RR}$ background, taken to be $X^{0,1}$-independent for simplicity, as follows:

$$
C_{1} \rightarrow C, \quad C_{\mu} \rightarrow C_{\mu 1} \quad(\mu \neq 1) .
$$

The world-volume action in dual language takes therefore the form

$$
\begin{aligned}
S_{\substack{\text { world } \\
\text { vol }}}=T_{(1)} & \int d^{2} \zeta \sqrt{1-\left(2 \pi \alpha^{\prime} \mathcal{F}_{01}\right)^{2}}+ \\
& +\mu_{(1)} \int d^{2} \zeta\left(C_{01}+2 \pi \alpha^{\prime} \mathcal{F}_{01} C\right)+\ldots
\end{aligned}
$$

in the physical parametrization $\zeta^{0,1}=X^{0,1}$.

Something surprising has happened here: from the simple point-particle Lagrangian, fixed completely by Poincare invariance, we are extracting seemingly non-trivial information about open-string gauge dynamics. What we are learning in particular is (i) that the effective Lagrangian for slowly-varying electromagnetic fields must be of the Born-Infeld type 33 , and (ii) that there are extra Wess-Zumino terms 35, 34, which when generalized appropriately include the anomaly-cancelling Green-Schwarz couplings 13 . Both of these key features follow directly from T-duality and Lorentz invariance. They also follow presumably from the requirement that one of the two ten-dimensional supersymmetries, broken spontaneously by the presence of the D-brane, be realized non-linearly on the world volume 32 . I suspect these two points of view are related though I am not sure of the details. There is finally another intriguing facet to the argument: the Born-Infeld action has a limiting electric field and a natural dissipation mechanism 37 to account for itf. In ( $\mathrm{T}$ )-dual language this would imply that the speed of light is a dynamical rather than a mere kinematic limit 24 , a conclusion befitting a fundamental theory of gravity.

I will stop here even though the fun is only starting! The interested reader will find many more details in Polchinski's nice review $\mathbb{E}$, and in the more than hundred papers on D-branes in hep-th this past year.

\footnotetext{
${ }^{c}$ To be sure, near the limiting field there is no real quantitative control of the dissipation rate.
} 


\section{Acknowledgments}

I thank the organizers for the invitation to speak in a very stimulating conference, and aknowledge partial support from the EEC grant CHRX-CT93-0340.

\section{References}

1. J. Polchinski, Phys. Rev. Lett. 75, 4724 (1995)

2. C.M. Hull and P.K. Townsend, Nucl. Phys. B 438, 109 (1995);

P.K. Townsend, Phys. Lett. B 350, 184 (1995)

3. E. Witten, Nucl. Phys. B 443, 85 (1995)

4. J. Polchinski, Lectures on D-Branes, in TASI 1996, hep-th/9611050;

J. Polchinski, S. Chaudhuri and C. Johnson, Notes on D-Branes, Lectures at ITP, hep-th/9602052.

5. J.H. Schwarz, Lectures on Superstring and M-theory Dualities, in TASI 1996, hep-th/9607201

6. M.R. Douglas, Superstring Dualities and the Small Scale Structure of Space, in Les Houches 1996, hep-th/9610041

7. P. Townsend, Four Lectures on M-theory, in Trieste 1996, hepth/9612121

8. S. Förste and J. Louis, Duality in String Theory, in this volume, hepth/9612192

9. M.J. Duff, Supermembranes, in this volume, hep-th/9611203

10. P. Aspinwall, K3 Surfaces and String Duality, hep-th/9611137.

11. J.M. Maldacena, Black Holes in String Theory, Princeton PhD thesis, hep-th/9607235

12. A. Hashimoto and I. Klebanov, Scattering of Strings from D-Branes, in Trieste 1996, hep-th/9611214

13. M.B. Green, J.H. Schwarz and E. Witten, Superstring Theory (Cambridge U. Press, 1987).

14. J.H. Schwarz, Phys. Lett. B 360, 13 (1995), and B364, 252 (1995)

15. E. Witten, Nucl. Phys. B 460, 335 (1996)

16. A. Strominger, Nucl. Phys. B 451, 96 (1995)

17. M. Bershadsky, V. Sadov and C. Vafa, Nucl. Phys. B 463, 398 (1996); M. Douglas and G. Moore, hep-th/9603167.

18. S. Ferrara, J. Harvey, A. Strominger and C. Vafa, Phys. Lett. B 361, 59 (1995);

S. Kachru and C. Vafa, Nucl. Phys. B 450, 69 (1995)

19. M.B. Green, Phys.Lett.B69, 89 (1977); B201, 42 (1988); B329, 435 (1994) 
20. M. Lüscher, K. Symanzik and P. Weisz, Nucl. Phys. B 173, 365 (1980);

O. Alvarez, Phys. Rev. D 24, 440 (1981);

J.F. Arvis, U. of Paris PhD thesis, 1983

21. J. Dai, R.G. Leigh and J. Polchinski, Mod.Phys.Lett. A4, 2073 (1989);

P. Horava, Phys. Lett. B 231, 251 (1989);

M.B. Green, Phys. Lett. B 266, 325 (1991)

22. A. Sagnotti, Non-Perturbative Quantum Field Theory, eds. G. Mack et al, (Pergamon Press) 521;

G. Pradisi and A. Sagnotti, Phys. Lett. B 216, 59 (1989);

M. Bianchi and A. Sagnotti, Phys. Lett. B 247, 517 (1990); Nucl. Phys. B 361, 519 (1991)

23. C.G. Callan, C. Lovelace, C.R. Nappi and S.A. Yost, Nucl. Phys. B 308, 221 (1988);

M. Li, Phys. Rev. D 54, 1644 (1996);

D. Polyakov, Nucl. Phys. B 468, 155 (1996)

24. C. Bachas, Phys. Lett. B 374, 37 (1996)

25. M.B. Green and M. Gutperle, hep-th/9604091;

G. Lifschytz, hep-th/9604156 and hep-th/9610125

26. M. Veltman, Methods in Field Theory, eds. R. Balian et al (North Holland,1975).

27. P.A.M. Dirac, Proc. Roy. Soc. A133 (1931);

R.I. Nepomechie, Phys. Rev. D 31, 1921 (1985);

C. Teitelboim, Phys. Lett. B 167, 63,69 (1986)

28. M. Douglas, hep-th/9604198;

M. Douglas, D. Kabat, P. Pouliot and S. Shenker, hep-th/9608024;

A. Sen, hep-th/9605150;

T. Banks, M. Douglas and N. Seiberg, hep-th/9605199

29. A. Strominger and C. Vafa, Phys. Lett. B 379, 99 (1996)

30. T. Banks, W. Fischler, S. Shenker and L. Susskind, hep-th/9610043

31. A. Giveon, M. Porrati and E. Rabinovici, Phys.Rep. 244, 77 (1994)

32. J. Hughes, J. Liu and J, Polchinski, Phys. Lett. B 180, 370 (1986);

J. Hughes and J. Polchinski, Nucl. Phys. B 278, 147 (1986);

J. Bagger and A. Galperin, hep-th/9608177

33. E.S. Fradkin and A. Tseytlin, Phys. Lett. B 163, 123 (1985);

R.G. Leigh, Mod.Phys.Lett.A4, 2767 (1989)

34. M.R. Douglas, hep-th/9512077

35. M. Li, Nucl. Phys. B 460, 351 (1996)

36. E. Bergshoeff, M. de Roo, M.B. Green, G. Papadopoulos and P.K.

Townsend, Nucl. Phys. B 470, 113 (1996);

E. Bergshoeff and M. de Roo, Phys. Lett. B 380, 265 (1996); 
E. Alvarez, J.L.F. Barbon and J. Borlaf, hep-th/9603089;

M.B. Green, C.M. Hull and P.K. Townsend, Phys. Lett. B 382, 65 (1996)

37. C. Bachas and M. Porrati, Phys. Lett. B 296, 77 (1992). 